\title{
TERAPIA NUTRICIONAL ENTERAL EM ADULTOS: NECESSIDADE ENERGÉTICA E PROTÉICA PRESCRITA VERSUS VOLUME ADMINISTRADO
}

\section{ENTERAL NUTRITIONAL THERAPY IN ADULTS: ENERGETIC AND PROTECTIVE NEEDED PRESCRIBED VERSUS ADMINISTERED VOLUME}

\author{
Maria Luiza Schoder ${ }^{1}$, Débora Regina Hendges Poletto Pappen ${ }^{2 \star}$ \\ ${ }^{1}$ Nutricionista, Centro Universitário Fundação Assis Gurgacz (FAG). ${ }^{2}$ Mestre. Docente do Curso de \\ Nutrição, Centro Universitário Fundação Assis Gurgacz (FAG). \\ * Autor correspondente: de poletto@hotmail.com, https://orcid.org/0000-0003-0643-0346
}

\begin{abstract}
RESUMO
A nutrição enteral deve ser indicada a pacientes com ingesta alimentar via oral insuficiente por três dias consecutivos ou pacientes impossibilitados de ingesta alimentar via oral. Entretanto, observa-se em muitos serviços que nem sempre todos os pacientes têm a prescrição de TNE adequada às suas necessidades, ou ainda, que a prescrição atende ao seu requerimento, porém, por inúmeros motivos o volume diário prescrito não é totalmente infundido. O objetivo da pesquisa foi averiguar se as prescrições dietoterápicas e o volume de dieta enteral administrado atendem ao requerimento energético e protéico de pacientes adultos hospitalizados em uso exclusivo de nutrição enteral. A técnica para seleção da amostragem utilizada foi retrospectiva, através de coleta de dados por meio da revisão do prontuário de pacientes oncológicos que utilizaram nutrição enteral no segundo semestre de 2013, foram coletados dados para amostra onde o paciente foi avaliado quanto à prescrição dietoterápica se o volume de nutrição enteral prescrito foi infundido completamente. Dos 38 prontuários analisados entre adultos (20-60) e idosos (>60), 10 eram do gênero feminino e 28 do gênero masculino. A idade média foi de 61,7 anos, sendo que $63,15 \%$ tinham 60 anos ou mais. Onde a TNE supriu apenas $70,3 \%$ das necessidades energéticas totais dos pacientes. A infusão da dieta enteral em unidades hospitalares ainda é um problema neste contexto, tendo em vista que muitos pacientes não recebem todo o seu requerimento energético e proteico, contribuindo para a desnutrição hospitalar e, piores desfechos clínicos.
\end{abstract}

Palavras-chave: Nutrição Enteral, Paciente Oncológico, Requerimento Calórico Proteico

ABSTRACT
Enteral nutrition should be indicated for patients with inadequate oral feeding intake for three consecutive days or patients unable to take oral food intake. However, it is observed in many services that not always all patients have the prescription of TNE adequate to their needs, or that the prescription meets their request, but for many reasons the prescribed daily volume is not fully infused. The aim of the study was to determine if the dietary prescriptions and the volume of enteral diet administered meet the energetic and protein requirements of adult patients hospitalized exclusively for enteral nutrition. The technique for selection of the sampling was retrospective, through data collection through the review of the medical records of oncology patients who used enteral nutrition in the second half of 2013, data were collected for a sample where the patient was evaluated for dietary prescription if the volume of enteral 
nutrition prescribed was completely infused. Of the 38 charts analyzed between adults (20-60) and elderly (>60), 10 were female and 28 male. The mean age was 61.7 years, and $63.15 \%$ were 60 years or older. The infusion of the enteral diet into hospital units is still a problem in this context, since many patients do not receive all their energy and protein requirements, contributing to the malnutrition and worse clinical outcomes.

Keywords: Enteral Nutrition, Cancer Patient, Protein Caloric Requirement

\section{INTRODUÇÃO}

As doenças crônicas são as principais causas de morte no mundo, dentre as quais o câncer tem se destacado por seu crescimento em todos os continentes. Nos países desenvolvidos e em desenvolvimento, encontra-se como a segunda e a terceira causa de morte, respectivamente (BENARROZ, 2009).

Segundo a Organização Mundial de Saúde (OMS), a distribuição de mortes por cânceres pelo mundo não é homogênea. A mortalidade total acarretada por cânceres foi de 12,6\% em 2000; $21,6 \%$ e 9,8\% nos países desenvolvidos e em desenvolvimento, respectivamente. Para os anos entre 1960 e 2000, os dados mostram um aumento de $15 \%$ para $25 \%$ de mortalidade nos países desenvolvidos. Já nos países em desenvolvimento, observaram-se taxas menores e crescentes, que alcançaram 6\% em 1985 e 9\% em 1997, com uma expectativa de aumentar, de 5,4 milhões em 2000, para 9,3 milhões em 2020, de acordo com projeções populacionais (GAROFOLO et al., 2014).

A presença do câncer altera, indubitavelmente, todos os aspectos da vida do indivíduo e pode acarretar profundas alterações no modo de viver habitual, conforme o comprometimento da capacidade e habilidade para execução de atividades de rotina. As alterações da integridade físico-emocional por desconforto, dor, desfiguração, dependência e perda da autoestima são relatadas por esses doentes que percebem a qualidade de suas vidas profundamente alterada, num curto período de tempo (MICHELONE, 2003).

O estado nutricional apresenta expressiva importância no tratamento do paciente oncológico. Com frequência estes pacientes cursam com déficits nutricionais decorrentes de efeitos colaterais da terapia antineoplásica, além de aumento do gasto energético pela doença de base e, menor ingestão alimentar, interferindo negativamente na tolerância a terapia empregada. A reduzida tolerância limita a dose e a eficácia do tratamento oncológico e resulta em toxicidade severa e diminuição dos efeitos desejados, podendo levar à morte (SANTOS, 2001).

A Terapia Nutricional Enteral (TNE) é definida como "alimento para fins especiais, com ingestão controlada de nutrientes, na forma isolada ou combinada, de composição definida ou estimada, especialmente formulada e elaborada para uso por sondas ou via oral, industrializada ou não, utilizada exclusiva ou parcialmente para substituir ou complementar a alimentação oral em pacientes desnutridos ou não, conforme suas necessidades nutricionais, em regime hospitalar, ambulatorial ou domiciliar, visando à síntese ou manutenção dos tecidos, órgãos ou sistemas" (BRASIL, 2000).

A TNE surge como uma possibilidade terapêutica de manutenção ou recuperação do estado nutricional, naqueles indivíduos que apresentarem o trato gastrintestinal integro para o processo digestório, mas com a ingestão oral parcial ou totalmente comprometida. A TNE precoce pode ser um importante fator na promoção da saúde, diminuição do estresse fisiológico e manutenção da imunidade. Nesse cenário, a escolha e a prescrição da Nutrição Enteral (NE) é complexa e implica 
conhecimento clinico e nutricional. Por isso, tão importante quanto à prescrição da TNE adequada as necessidades do paciente, é a certeza de que o paciente efetivamente receberá o volume prescrito (ASSIS et al., 2010).

Atualmente, a Agência Nacional de Vigilância Sanitária (ANVISA) regulamenta a formação de Equipe Multidisciplinar de Terapia Nutricional (EMTN), obrigatória nos hospitais brasileiros. Essa regulamentação é regida pelas portarias 272 (Regulamento Técnico de Terapia de Nutrição Parenteral) e 337 (Regulamento Técnico de Terapia de Nutrição Enteral). Fazem parte das atribuições da EMTN: definir metas técnicoadministrativas, realizar triagem e vigilância nutricional, avaliar o estado nutricional, indicar terapia nutricional e metabólica, assegurar condições ótimas de indicação, prescrição, preparação, armazenamento, transporte, administração e controle dessa terapia; educar e capacitar a equipe; criar protocolos, analisar o custo e o benefício e traçar metas operacionais da EMTN (LEITE et al., 2005).

Alterações da motilidade gastrintestinal são comuns em pacientes hospitalizados em uso de NE e, podem variar dependendo de sua condição clínica e seu estado nutricional (BTAICHE et al., 2010). Dentre as alterações comuns, a diarreia, distensão abdominal, náuseas e vômitos são as complicações de maior frequência em pacientes com terapia nutricional enteral (LOPEZ-HERCE, 2009; KOZAR et al., 2002).

Estas complicações quando não notificadas podem trazer inúmeras complicações ao paciente, dentre eles, infecção e problemas de pele (como úlceras por pressão), perda de eletrólitos, desidratação, desnutrição e hipoalbuminemia (COUTO et al, 1998), levando ao prolongamento da permanência hospitalar, o que contribui para maiores índices de morbimortalidade, além da elevação dos custos (CRESCI, 2001; LOPEZ-HERCE, 2009; BTAICHE et al., 2010).

Sabe-se que o adequado manejo nutricional pode interferir na maioria dos sintomas apresentados acima, portanto, a adequada e efetiva comunicação ao Serviço de Nutrição e Dietética (SND), contribui para que o paciente possa de fato receber o aporte calórico-proteico adequado as suas necessidades, ao invés da precipitada e errônea suspensão da nutrição enteral (COUTO et al, 1998).

Este estudo teve por objetivo avaliar se as prescrições atendem as necessidades energéticas e proteicas dos pacientes internados $e$, ainda, se as prescrições são integralmente administradas, é de extrema importância para avaliar quais as dificuldades na estimativa das necessidades de cada paciente e, os motivos apontados para a não administração do volume de nutrição enteral prescrito.

\section{METODOLOGIA}

Este estudo caracterizou-se como pesquisa de campo, exploratória, com abordagem qualitativa e quantitativa. A técnica para seleção da amostragem utilizada foi retrospectiva.

A coleta de dados foi realizada em formulário específico, elaborado pela própria pesquisadora, por meio da revisão do prontuário de pacientes oncológicos que utilizaram nutrição enteral no segundo semestre de 2013. Para esta etapa da pesquisa, não se faz necessária nenhuma infraestrutura específica. Onde foram revisados 52 prontuários, sendo 14 destes excluídos.

Os critérios de inclusão foram: pacientes que utilizaram nutrição enteral exclusiva ou associada à outra forma de terapia nutricional (oral ou parenteral), internados na enfermaria ou na Unidade de Terapia Intensiva no segundo semestre de 2013. 
Os critérios de exclusão foram: Pacientes que não utilizaram nutrição enteral ou que estavam em jejum ou apenas com nutrição via oral internados na enfermaria ou na Unidade de Terapia Intensiva no segundo semestre de 2013. Pacientes pediátricos que utilizaram nutrição enteral exclusiva ou associada à outra forma de terapia nutricional (oral ou parenteral), internados na enfermaria ou na Unidade de Terapia Intensiva no segundo semestre de 2013.

Para avaliar o diagnóstico do estado nutricional, foi divido dois grupos, 0 primeiro grupo era composto por pacientes com faixa etária de 20 a 60 anos, já o segundo grupo representava os idosos com 60 anos ou mais. Foi utilizado o Índice de Massa Corporal (IMC) de acordo com a OMS (1997) sendo adotada a seguinte classificação para adultos: Baixo Peso: IMC < 18,5 kg/m², Eutrófico: IMC $\geq 18,5$ e < $24,9 \mathrm{~kg} / \mathrm{m}^{2}$, Sobrepeso: IMC $\geq 25$ e $<29,9 \mathrm{~kg} / \mathrm{m}^{2}$, Obesidade: $\geq 30 \mathrm{~kg} / \mathrm{m}^{2}$. Para os idosos foi adotada a classificação de LIPSCHITZ (1994): Magreza: $<22 \mathrm{~kg} / \mathrm{m}^{2}$, Eutrófico: $\geq 22$ e $<27 \mathrm{~kg} / \mathrm{m}^{2}$, Sobrepeso: $\geq 27 \mathrm{~kg} / \mathrm{m}^{2}$.

Através da verificação de prontuários dos pacientes que estavam hospitalizados foram coletados dados para amostra onde o paciente foi avaliado quanto à prescrição dietoterápica, para avaliar se esta atende as suas necessidades energéticas e ebem como, se a prescrição foi atendida plenamente, ou seja, se o volume de nutrição enteral prescrito foi infundido completamente.

Para verificar a quantidade de volume prescrito e administrado, foi necessário fazer o cálculo através da dieta prescrita, onde foi conferido através da rotulagem de cada dieta prescrita conforme necessidade de cada paciente, a densidade calórica e proteica, sendo realizado com a regra de três, sendo esta uma fórmula onde tem quatro valores, mas temos conhecimento apenas de três resultados. $E$ também para finalizar foi realizado adequação calórica e proteica, onde era feito através da subtração do valor prescrito ao valor administrado, sendo assim obtendo o valor e depois convertendo este mesmo resultado em porcentagem, para melhor visualização.

Os prontuários dos pacientes foram analisados somente durante o período em que utilizaram nutrição enteral, a partir do momento em que ocorre-se caso de óbito, o paciente foi retirado do estudo, sendo este seu último dia de avaliação.

\section{RESULTADOS E DISCUSSÃO}

Dos 38 prontuários analisados entre adultos (20-60) e idosos ( $>60), 10$ eram do gênero feminino e 28 do gênero masculino. A idade média foi de 61,7 anos, sendo que 63,15 \% tinham 60 anos ou mais. A média de internação foi de 18 dias.

A Tabela 1 apresenta a distribuição dos pacientes em relação ao estado nutricional e a faixa etária. Em relação ao estado nutricional observou-se que a maioria dos pacientes com 60 anos ou mais, apresentaram que 50\% eram eutróficos, e 16,6\% apresentava-se com baixo peso e 33,3\% destes pacientes idosos estavam sobrepeso. E os pacientes de 20 a 60 anos se encontravam tanto peso adequado e sobrepeso, com um total de $42,8 \%$, respectivamente. E com estado nutricional de obesidade grau I e II apresentaram um total de 7,14\%.

A desnutrição hospitalar vem sendo estudada há décadas, e relatos na literatura científica têm indicado que 20 a $50 \%$ dos pacientes hospitalizados apresentam algum grau de desnutrição (CAPANELLA, 2008). Segundo Campos et al. (2006), este percentual torna-se ainda mais elevado quando se trata de pacientes em nutrição enteral. Os mesmos autores verificaram em seu estudo, uma prevalência de 
$54,8 \%$ de desnutrição. Onde na atual pesquisa o resultado encontrado foi de $16,6 \%$ apenas, e em pacientes com 60 anos ou mais.

Tabela 1. Distribuição dos pacientes em relação ao estado nutricional e classificação nos 38 protocolos analisados.

\begin{tabular}{|c|c|c|c|c|c|c|c|c|c|c|c|c|c|}
\hline \multirow{2}{*}{ FAIXAETÁRIA } & \multirow{2}{*}{$\mathrm{N}^{0}$} & \multicolumn{2}{|c|}{ BAIXO PESO } & \multicolumn{2}{|c|}{ PESO ADEQUADO } & \multicolumn{2}{|c|}{ SOBREPESO } & \multicolumn{2}{|c|}{ OBESIDADEI } & \multicolumn{2}{|c|}{ OBESIDADE II } & \multicolumn{2}{|c|}{ OBESIDADE III } \\
\hline & & $\mathrm{N}$ & $\%$ & $\mathrm{~N}$ & $\%$ & $\mathrm{~N}$ & $\%$ & N & $\%$ & $\mathrm{~N}$ & $\%$ & $\mathrm{~N}$ & $\%$ \\
\hline $20-60$ & 14 & 0 & 0 & 6 & 42,8 & 6 & 42,8 & 0 & 0 & 1 & 7,14 & 1 & 7,14 \\
\hline 60 ou mais & 24 & 4 & 16,6 & 12 & 50 & 8 & 33,3 & 0 & 0 & 0 & 0 & 0 & 0 \\
\hline
\end{tabular}

Acuna e Cruz (2004), um estudo confirmaram que os idosos são mais propensos à desnutrição, em decorrência de alterações fisiológicas e sociais, como, uso de polifármacos; problemas relacionados à alimentação (comprometendo a mastigação e deglutição); depressão e alterações da mobilidade, com dependência funcional.

Segundo Lamb et al. (2003), pacientes idosos, normalmente, utilizam o suporte nutricional enteral com mais frequência, como observado na atual pesquisa, já que esse grupo é hospitalizado com mais cautela.

Em um estudo realizado por Detregiachi et al. (2011), com amostragem de 59 pacientes, ele encontrou entre os pacientes avaliados uma média de IMC de 21,4 $\pm 6,4$ $\mathrm{kg} / \mathrm{m} 2$, não havendo diferença entre os sexos. A distribuição do diagnóstico nutricional, segundo este dado, foi de $54,2 \%$ dos pacientes com baixo peso, $32,2 \%$ eutróficos e 13,6\% apresentavam excesso de peso.

Já Isidro et al. (2012), em seu estudo com 32 pacientes, encontrou em relação ao estado nutricional, Uma média de $40,6 \%$, um total de pacientes, estavam desnutridos segundo o IMC.

De acordo com a figura 1, observa-se que $57,9 \%$ dos pacientes estudados 0 motivo para suspensão da nutrição enteral foi por conta de óbito, um pouco mais que a maioria. Em 18,4\% dos casos o paciente passou para dieta via oral, havendo uma melhora no seu estado nutricional. Já em relação à distensão abdominal encontra-se $10,5 \%$. A diarreia foi presente somente em $7,9 \%$, seguido por 2,6\% recusa do paciente ou até mesmo em alguns casos da família do mesmo e com o mesmo valor $2,6 \%$ a opção outros, onde neste item pode se encaixar rompimento da dieta por motivo de exames, alguns procedimentos como drenagem de líquidos, como bile ou sangue. 


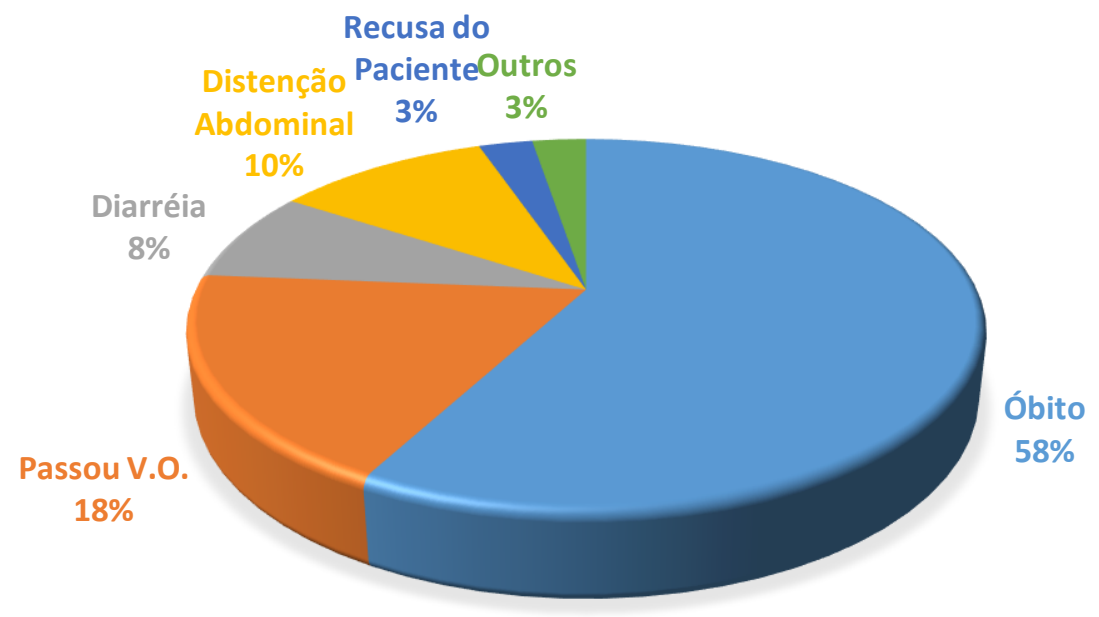

Figura 1. Motivos encontrados para suspensão da dieta enteral em pacientes hospitalizados no segundo semestre de 2013.

Assis et al. (2010), em seu estudo ele observou que os motivos mais frequentemente relatados nos prontuários para interrupção da dieta foram: presença de náuseas e vômitos (15,2\%), distensão abdominal (14,4\%), constipação $(8,8 \%)$, complicações clínicas $(14,4 \%)$, realização de procedimentos diagnósticos $(41,6 \%)$ e transição para alimentação oral $(5,6 \%)$.

No estudo feito por Piovacari et al. (2007) entra as principais intercorrências que impedem a infusão da dieta prescrita foram $39 \%$ jejum para procedimento, $21 \%$ por falta de registro em prontuário, $8 \%$ de náuseas, vômitos e refluxo e apenas $7 \%$ de diarreia.

Já em um estudo realizado por Agudelo et al. (2011), com uma população alvo de 419 pacientes avaliados, 380 receberam Nutrição Enteral (NE) e 39 Nutrição Parenteral (NP). A complicação de maior incidência de NE foi o resíduo gástrica elevada $(24,2 \%)$, seguido de diarreia (14\%) e a remoção do cateter $(6,6 \%)$.

$\mathrm{Na}$ pesquisa realizada por Isidro et al. (2012), dos pacientes analisados, 26 deles $(81,3 \%)$ apresentaram alguma intercorrência que levou à suspensão da TNE. Destes, 13 pacientes $(50 \%)$ recebiam a dieta no pré-operatório, período no qual apenas um $(7,1 \%)$ não apresentou fatores que interferissem na dieta planejada. No pós-operatório, apenas cinco $(27,8 \%)$ não apresentaram nenhuma intercorrência, demonstrando não haver diferença estatística $(p=0,153)$ quanto à ocorrência de causas de suspensão da dieta quando comparamos o período em foi utilizada a TNE. As principais causas de interrupção da dieta encontradas neste trabalho, foram no pré-operatório $84,6 \%$ jejum para procedimentos, $30,8 \%$ dor e distensão abdominal e $23 \%$ saída da sonda, também foi encontrado o mesmo resultado $7,69 \%$ tanto para diarreia, náuseas/vômitos e recusa do paciente. Já no pós-operatório encontraram $38,5 \%$ náuseas/vômitos, 30,8 \% saída da sonda, $23 \%$ dor e distensão abdominal, $15,4 \%$ tanto jejum para procedimentos quanto retorno gástrico, e $7,69 \%$ casos de diarreia e o mesmo valor recusa do paciente.

A figura 2 apresenta a quantidade de dieta enteral administrada e a quantidade que faltou em relação a dieta prescrita, através de uma média total dos 38 pacientes analisados. Onde podemos observar que $70,3 \%$ da dieta foi administrada e $29,7 \%$ foi 
o que faltou para completar a quantidade prescrita. Em relação tanto com a densidade calórica e a densidade proteica.

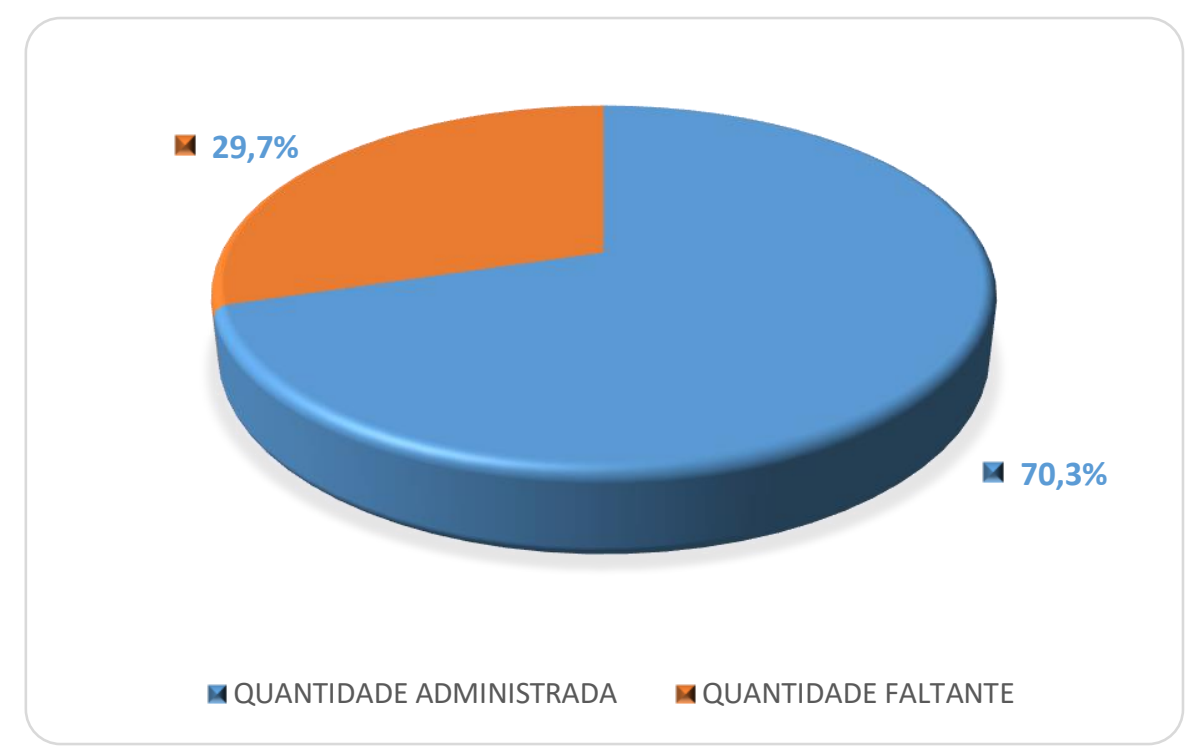

Figura 2. Média da porcentagem administrada em relação a prescrita calórica e proteica.

De acordo com Assis e demais autores (2010), onde realizaram um estudo com 85 pacientes em uso exclusivo de NE, onde permaneceram em média 29,5 dias. Neste período, evidenciou-se que, em média, os pacientes receberam menos volume ($428 \pm 243 \mathrm{ml} / \mathrm{dia})$, calorias $(-665 \pm 412 \mathrm{Kcal} / \mathrm{dia})$ e proteínas $(-30 \pm 19 \mathrm{~g}$ de proteína/dia) do que o prescrito, representando cerca de $40 \%$ de redução. No atual estudo podemos observar que os 38 pacientes analisados do total prescrito os mesmos receberam um total de 70,3\%, em média.

Já em um estudo realizado por Teixeira et al. (2006), onde foram acompanhados 33 pacientes, com idade média de 57 anos (variando entre 18 e 85 anos) e $58 \%$ do sexo masculino. Foi observado que a quantidade diária de calorias e proteínas prescritas foram, respectivamente, de 1715,7 $\pm 250,8 \mathrm{kcal}(26,1 \pm 3,7$ $\mathrm{kcal} / \mathrm{kg})$ e $68,6 \pm 10,1 \mathrm{~g}(1,04 \pm 0,1 \mathrm{~g} / \mathrm{kg})$. Esses valores estão bem próximos da média das necessidades calculadas, o que indica uma adequação entre o prescrito e as necessidades do paciente.

De acordo com estudo feito por Detregiachi et al., (2011) a necessidade energética (GET) média foi de $1635 \pm 417,8 \mathrm{Kcal} / \mathrm{dia}$. A média de energia prescrita foi de $1045 \mathrm{kcal} / \mathrm{dia}$ e a ofertada, a partir do quarto dia de implementação da TNE, foi de $1035 \pm 422 \mathrm{Kcal} / \mathrm{dia}$. Verificou-se uma diferença significativa entre a necessidade e a oferta energética por meio da NE $(p<0,05)$ assim como entre ao valor energético necessário e o prescrito $(p<0,05)$, ficando ambos aquém da necessidade.

Na pesquisa elaborada por Isidro et al. (2012), a diferença entre o administrado e o prescrito foi significativa, com um déficit de $105,9 \mathrm{Kcal} /$ dia e de $5,5 \mathrm{~g}$ de proteína/dia $(p<0,0001)$. Do total, 19 pacientes $(59,4 \%)$ estavam adequados $(\geq 90 \%)$ quanto a calorias e $18(56,2 \%)$ quanto a proteínas.

Em um estudo realizado por Elpern et al. (2004), onde ele estudou 39 pacientes durante 279 dias com nutrição enteral, em que estes pacientes receberam $64 \%$ da meta, uma média de $669 \mathrm{kcal}$ a menos do necessário diariamente. 
Porém em um estudo realizado por Piovacari et at (2007), com uma amostragem de 22 mulheres e 21 homens, observou que 82,7\% da quantidade prescrita foi infundida quando ocorreu alguma intercorrência, como jejum para procedimentos, falta de registro no prontuário, diarreia, náuseas, vômitos e refluxo. Entretanto a média de dieta infundida quando não houve nenhuma intercorrência foi de $103,7 \%$.

\section{CONCLUSÃO}

Os resultados deste estudo indicam que a infusão da dieta enteral em unidades hospitalares ainda é um problema neste contexto, tendo em vista que muitos pacientes não recebem todo o seu requerimento energético e proteico, contribuindo para a desnutrição intra hospitalar e, piores desfechos clínicos.

Onde observamos que os pacientes receberam apenas $70,3 \%$ da dieta prescrita, nos mostram a necessidade de adoção de mecanismos de vigilância clínica afim de assegurar que um melhor manejo nutricional seja instituído a pacientes hospitalizados em uso de TNE.

Sugere-se que a presença de uma equipe multidisciplinar em terapia enteral, a realização de treinamentos específicos com a equipe e a elaboração e aplicação de um protocolo de ação sejam medidas que possam minimizar as intercorrências encontradas.

Podemos notar também neste estudo os principais motivos para suspensão da dieta enteral, onde encontramos casos de óbito, diarreia, distensão abdominal entre outros, Assim com auxilio também de uma equipe bem treinada, havendo possibilidade de diminuir estes quadros.

Lembrando que com a conscientização de toda a equipe isso é possível, pois a prescrição da TN é médica, as adequações são do nutricionista e, a administração da enfermagem, fazendo com que isto vire rotina, melhorando a qualidade de vida e até mesmo ocorrendo uma diminuição de mortalidade e reduzindo custos ao hospital.

\section{REFERÊNCIAS}

ACUNA K., CRUZ T. Avaliação do estado nutricional de adultos e idosos e situação nutricional da população brasileira. Arquivos Brasileiros de Endocrinologia e Metabolismo. v.48, n.3, p. 345-61, 2004.

AGUDELO G. M. et al., Incidencia de complicaciones del soporte nutricional en pacientes críticos: estudio multicêntrico. Nutrição Hospitalaria. V.26, n. 3, p. 537545, 2011.

ASSIS M. C. S., SILVA S. M. R., LEAES D. M., NOVELLO C. L., SILVEIRA C. R. M., MELLO E. D., BEGHETTO M. Nutrição enteral: diferenças entre volume, calorias e proteínas prescritos e administrados em adultos. Revista Brasileira de Terapia Intensiva. V.22, n. 4, p.346-350, 2010.

BENARROZ M. O., FAILLACE G. B. D., BARBOSA L. A. Bioética e nutrição em cuidados paliativosoncológicos em adultos. Cadernos de Saúde Pública, v. 25, n.9, p.1875-1882, 2009. 
BRASIL. Resolução n. 63 , de 6 de julho de 2000. Aprova o Regulamento Técnico para fixar os requisitos mínimos exigidos para a Terapia de Nutrição Enteral.

BTAICHE I. F., CHAN L. N., PLEVA M., KRAFT M. D. Critical illness, gastrointestinal complications, and medication therapy during enteral nutrition in critically ill adult patients. Nutrition in Clinical Practice. V.25, p.32-49, 2010.

CAMPOS D. J., SILVA A. F. F., SOUZA M. H. Otimização do fornecimento calórico protéico na terapia intensiva nutricional em unidade de terapia intensiva com o uso de protocolo. Revista Brasileira de Nutrição Clínica v.21, n.1, p.2-5, 2006.

CAPANELLA, L. C. A., et al. Terapia Nutricional Enteral: A dieta prescrita é realmente infundida? Revista Brasileira de Nutrição Clínica v.23, n.1, p21-5, 2008.

COUTO C. M. F., Sales TRA, Torres HOG, Carvalho EB. Avaliação de Hipoalbuminemia como fator de risco para diarreia associada à nutrição enteral. Revista Brasileira de Nutrição Clínica v.13, n.1, p. 3-20,1998

CRESCI G. A. The use of probiotics with the treatment of diarrhea. Nutr Clin Pract. V.16, p.30-4, 2001

DETREGIACHI C. R. P., QUESADA K. R., MARQUES D. E. Comparação entre as necessidades energéticas prescrita e administradas a pacientes em terapia nutricional enteral. Medicina (Ribeirão Preto) v.44, n.2, p. 177-84. 2011

ELPERN E. H., STUTZ L., PETERSON S., GURKA D. P., SKIPPER A., Outcomes Associated With Enteral Tube Feedings in a Medical Intensive Care Unit. American Journal of Critical Care, v.13, p.221-227, 2004.

GAROFOLO A., AVESAN C. M., CAMARGO K. G., BARROS M. E., SILVA S. R. J., TADDEI J. A. A. C., SIGULEM D. M. Dieta e câncer: Um enfoque epidemiológico. Revista de Nutrição, v.17, n.4, p.491-505, 2004.

ISIDRO M. F., LIMA D. S. C. Adequação calórico protéica da terapia nutricional enteral em pacientes cirúrgicos. Elsevier Editora Ltda 2012.

KOZAR R. A., MCQUIGGAN M. M., MOORE E. E., KUDSK K., JURKOVICH G., MOORE F. A. Postinjury enteral tolerance is reliably achieved by a standardized protocol. J Surg Rev. V. 104, p. 70-5, 2002.

LAMB A. T., SCHMIDT K. H. H., VIEIRA J. M. Perfil dos pacientes recebendo suporte nutricional. Rev Nutr Pauta. V. 11, n. 62, p.18-21, 2003.

LEITE H. P., CARVALHO W. B., MENESES J. F., Atuação da equipe multidisciplinar na terapia nutricional de pacientes sob cuidados intensivos. Revista de Nutrição, v. 18, n. 6, p. 777-784, 2005.

LOPEZ-HERCE J. Gastrointestinal complications in critically ill patients: what differs between adults and children. Current Opinion in Clinical Nutrition and Metabolic Care. V.12, p.180-5, 2009. 
MICHELONE A. P. C., SANTOS V. L. C. G. Qualidade de vida de adultos com câncer colorretal com e sem ostomia. Rev Latino-am Enfermagem, v.12, n.6, p.875-83, 2004.

PIOVACARI S. M. F., SILVA M. F. et al. Terapia Nutricional Enteral em Sistema Fechado. Volume Prescrito x Volume Infundido. Serviço de Nutrição, Hospital Israelita Albert Einstein. 2007

SANTOS H. S., CRUZ W. M. S. A Terapia Nutricional com Vitaminas Antioxidantes e o Tratamento Quimioterápico Oncológico. Revista Brasileira de Cancerologia, v. 47, n.3, p. 303-08, 2001.

TEIXEIRA A. C. C., CARUSO L., SORIANO F. G. Terapia Nutricional Enteral em Unidade de Terapia Intensiva: Infusão Versus Necessidades. Revista Brasileira de Terapia Intensiva. v. 18 N. 4, 2006. 